\title{
The influence of nutritional status on the incidence of postoperative complications in patients following distal pancreatectomy
}

\author{
Beata Jabłońska, Paweł Lampe, Sławomir Mrowiec \\ Department of Digestive Tract Surgery, Medical University of Silesia, Katowice, Poland
}

Gastroenterology Rev 2020; 15 (1): 65-75

DOI: https://doi.org/10.5114/pg.2019.86806

Key words: distal pancreatectomy, postoperative complications, nutritional status, malnutrition, prognostic nutritional index.

Address for correspondence: Beata Jabłońska, Department of Digestive Tract Surgery, University Hospital of the Medical University
of Silesia, 14 Medyków St, 40-752 Katowice, Poland, phone/fax: +48 3278942 51, e-mail: bjablonska@poczta.onet.pl

\begin{abstract}
Introduction: Malnutrition is a common problem in hospitalised patients. The immunological, inflammatory, and nutritional status of patients significantly influences the postoperative outcome.

Aim: To assess and analyse the influence of the nutritional status on postoperative complications in patients following distal pancreatectomy.

Material and methods: The analysis included 50 patients operated in a large centre of gastrointestinal surgery. The clinicopathological parameters were analysed, and the nutritional status was assessed. The prognostic nutritional index (PNI) was calculated as $10 \times$ serum albumin $(\mathrm{g} / \mathrm{dl})+0.005 \times$ total lymphocyte count (per $\left.\mathrm{mm}^{3}\right)$. The immunological parameters, neutrophil/ lymphocyte ratio (NLR), platelet/lymphocyte ratio (PLR), and lymphocyte/monocyte ratio (LMR) were calculated. Patients were divided into two groups according to the presence of early postoperative complications: those without postoperative complications and those with postoperative complications.

Results: Early postoperative complications were observed in 15 (30.0\%) patients undergoing distal pancreatectomy. Postoperative pancreatic fistula (POPF) was the most frequent complication noted in 11 (22\%) patients. Significantly higher Nutritional Risk Screening (NRS) $2002(p=0.005)$ and lower PNI (median value: 56 vs. 41, $p=0.0003$ ) were noted in patients with postoperative complications. In laboratory results, the significantly lower total lymphocyte count (median value: 2.4 vs. 1.4 per $\mathrm{mm}^{3}, p=0.01$ ) and serum level of albumin (median value: $4.7 \mathrm{vs} .3 .3 \mathrm{~g} / \mathrm{dl}, p=0.0003$ ) were noted in the complications group.

Conclusions: Nutritional status significantly influences the incidence of postoperative complications in patients following distal pancreatectomy. Assessment of nutritional status using PNI calculation should be the standard management of patients before surgical treatment.
\end{abstract}

\section{Introduction}

Distal pancreatectomy is a serious surgical procedure that is associated with a low mortality rate $(<5 \%)$ but a high percentage of morbidity, with postoperative complications ranging from $22.7 \%$ to $57 \%$ in high-volume pancreatic surgical centres [1-6].

Malnutrition is a common problem in hospitalised patients. According to the literature, $30 \%$ to $50 \%$ of hospitalised patients are malnourished. Malnutrition negatively influences patients' prognosis and quality of life. Proper assessment of nutritional status and preoperative nutritional intervention can help to decrease the risk of postoperative complications. In order to assess the nutritional status, both objective and subjective criteria are used, including various anthropometric, clinical, and biochemical parameters [7, 8].

It is known that the immunological, inflammatory, and nutritional status of patients significantly influences the postoperative outcome. In the literature there are a lot of studies regarding the significance of nutritional status in patients following gastric, bowel, and proximal pancreatic resections [9-15]. However, to our knowledge, there are not many reports presenting the impact of nutritional status on postoperative complications in patients following distal pancreatectomy. 


\section{Aim}

The aim of the study was the assessment of nutritional status in patients undergoing distal pancreatectomy using selected anthropometric, clinical, and biochemical parameters and analysis of the influence of nutritional status on the incidence of postoperative complications.

\section{Material and methods}

\section{Patients}

The analysis included 50 patients undergoing distal pancreatectomy in a large gastrointestinal surgery centre. Assessment of nutritional status was performed in patients at the time of admission to hospital, prior to surgical treatment. There were 21 (42\%) men and

Table I. The patients' clinicopathological characteristics

\begin{tabular}{|c|c|}
\hline Parameter & Value \\
\hline \multicolumn{2}{|l|}{ Demographic characteristics: } \\
\hline Age [years] & $57.34 \pm 12.07(30-80)$ \\
\hline Male/female & $21(42.00 \%) / 29(58.00 \%)$ \\
\hline BMI $\left[\mathrm{kg} / \mathrm{m}^{2}\right]$ & $26.43 \pm 5.69(18.16-52.44)$ \\
\hline NRS 2002 [points] & $0.64 \pm 1.08(0-4)$ \\
\hline ASA & $2.23 \pm 0.53(1-3)$ \\
\hline \multicolumn{2}{|l|}{ Tumour location: } \\
\hline Trunk & $10(20.00 \%)$ \\
\hline Tail & $23(46.00 \%)$ \\
\hline Trunk and tail & $17(34.00 \%)$ \\
\hline Tumour diameter $[\mathrm{cm}]$ & $4.28 \pm 3.17(0.6-19)$ \\
\hline \multicolumn{2}{|l|}{ Histopathological type: } \\
\hline Adenocarcinoma & $11(14.67 \%)$ \\
\hline Anaplastic carcinoma & $1(1.33 \%)$ \\
\hline Neuroendocrine tumour & $19(24.34 \%)$ \\
\hline Metastatic renal cell carcinoma & $4(5.33 \%)$ \\
\hline Serous cystic neoplasm & $7(9.33 \%)$ \\
\hline $\begin{array}{l}\text { Intraductal papillary mucinous } \\
\text { neoplasm }\end{array}$ & $2(2.67 \%)$ \\
\hline Mucinous cystic neoplasm & $1(1.33 \%)$ \\
\hline Metastatic melanoma & $1(1.33 \%)$ \\
\hline Intrapancreatic accessory spleen & $1(1.33 \%)$ \\
\hline Chronic pancreatitis & $4(5.33 \%)$ \\
\hline \multicolumn{2}{|l|}{ Surgical procedure/resection: } \\
\hline Tail & $13(26.00 \%)$ \\
\hline Trunk and tail & 37 (74.00\%) \\
\hline
\end{tabular}

29 (58\%) women, with a mean age of $57.34 \pm 12.07$ (30-80) years in the analysed group. Inclusion criteria comprised: pancreatic pathology (tumour) requiring surgical removal (distal pancreatectomy) and age $>18$ years. The exclusion criterion comprised: incomplete demographic and clinical data. The University Ethics Committee decided that for this type of study formal consent was unnecessary. Written, informed consent was obtained from all participants.

\section{Study design}

Information regarding deterioration of nutritional status, body weight before disease and current body weight, loss of body weight and food intake since the onset of disease, comorbidities (arterial hypertension, ischaemic heart disease, diabetes mellitus), and smok-

\begin{tabular}{|c|c|}
\hline Parameter & Value \\
\hline \multicolumn{2}{|l|}{ Splenectomy: } \\
\hline No & $10(20.00 \%)$ \\
\hline Yes & $40(80.00 \%)$ \\
\hline Intraoperative blood loss [ml] & $\begin{array}{l}633.33 \pm 544.90 \\
(0.00-2100.00)\end{array}$ \\
\hline \multicolumn{2}{|l|}{ Blood transfusion: } \\
\hline No & $44(88.00 \%)$ \\
\hline Yes & $6(12.00 \%)$ \\
\hline Operation duration [h] & $3.48 \pm 51.28(1.2-6.5)$ \\
\hline Total hospitalization duration [days] & $12.60 \pm 7.08(6-44)$ \\
\hline $\begin{array}{l}\text { Postoperative hospitalisation } \\
\text { duration [days] }\end{array}$ & $9.82 \pm 6.84(4-41)$ \\
\hline \multicolumn{2}{|l|}{ Arterial hypertension: } \\
\hline No & $33(76.00 \%)$ \\
\hline Yes & $17(34.00 \%)$ \\
\hline \multicolumn{2}{|l|}{ Ischaemic heart disease: } \\
\hline No & $46(90.00 \%)$ \\
\hline Yes & $4(8.00 \%)$ \\
\hline \multicolumn{2}{|l|}{ Diabetes mellitus: } \\
\hline No & $43(36.00 \%)$ \\
\hline Yes & $7(14.00 \%)$ \\
\hline \multicolumn{2}{|l|}{ Smoking: } \\
\hline No & $40(80.00 \%)$ \\
\hline Yes & $10(20.00 \%)$ \\
\hline \multicolumn{2}{|l|}{ Postoperative complications: } \\
\hline No & $35(70.00 \%)$ \\
\hline Yes & $15(30.00 \%)$ \\
\hline
\end{tabular}

Values are presented as means and standard deviations. BMI - body mass index, NRS 2002 - Nutritional Risk Score 2002, ASA - American Society of Anaesthesiologists. 
ing was collected. On admission, the height and weight were measured, and laboratory blood tests were performed. The selected blood count parameters (haemoglobin, total white blood cell, lymphocyte, neutrophil, monocyte, platelet counts) and biochemical parameters (serum total protein, albumin, and C-reactive protein (CRP)) were analysed. The body mass index (BMI) was calculated. The nutritional risk according to NRS 2002 (Nutritional Risk Screening 2002) by the European Society of Parenteral and Enteral Nutrition (ESPEN) was assessed [16, 17]. The immunological parameters, neutrophil/lymphocyte ratio (NLR), platelet/lymphocyte ratio $(P L R)$, and lymphocyte/monocyte ratio $(L M R)$, were calculated [18]. Onodera's nutritional prognostic index (PNI) was calculated based on the serum albumin concentration and total lymphocyte count in the peripheral blood using the following formula: $10 \times$ level of albumin $(\mathrm{g} / \mathrm{dl})+0.005 \times$ total lymphocyte count $\left(/ \mathrm{mm}^{3}\right)[19]$. The patients were divided into two groups according to the absence (group 1) or presence (group 2) of postoperative complications. Clinicopathological factors and laboratory parameters were compared between the two groups.

\section{Ethics approval and consent to participate}

The University Ethics Committee decided that for this type of study formal consent was unnecessary. Written, informed consent was obtained from all participants.

\section{Statistical analysis}

Comparison between groups was performed using Student's $t$-test, the $\chi^{2}$ test, and the Mann-Whitney $U$ test. The Shapiro-Wilk test was used to check for normality of the distribution. A $p$-value of $<0.05$ was considered statistically significant. The statistical analyses were performed using Statistica ${ }^{\circledR}$ software, version 13.0 (StatSoft).

\section{Results}

The general clinical characteristics of 50 patients are presented in Table I. In the analysed group, most frequent pathology (tumour) was located within the pancreatic tail $(23 ; 46 \%)$. Neuroendocrine tumours were the most frequent $(19 ; 24.34 \%)$ histopathological types. In most (37 cases; 74\%), resection of the pancreatic tail and trunk was performed. Distal pancreatectomy involving splenectomy was performed in 40 (80\%) patients. The values of laboratory tests are presented in Table II. The values of PNI ranged from 33 to 67.5 with a mean value 51.34 .

Postoperative complications were noted in 15 (30\%) patients. They are presented in Table III. Postoperative pancreatic fistula (POPF) was the most frequent complication $(11 ; 22 \%)$. The pancreatic fistula of type B was the most frequently recorded in the analysed group; it was observed in 6 patients (12\% of all complications and $54.54 \%$ of all pancreatic fistulas). Clinically relevant POPF (type B and C) was noted in seven (14\%) patients. The POPF was defined and classified according to International Study Group on Pancreatic Fistula [20]. There were three relaparotomies in the analysed group. The first one was performed on the $17^{\text {th }}$ postoperative day due to a retroperitoneal abscess. Drainage of the abscess was made. The second one was performed on the fourth postoperative day due to intraperitoneal bleeding caused by anticoagulant drugs administrated as treatment for a pulmonary embolism. Packing was performed. The mortality rate was $0 \%$. There were three rehospitalisations (6\%) due to intraabdominal fluid collections that were treated conservatively.

The analysed patients were divided into groups according to the presence or absence of early postoperative complications (group 1 - without complications, group 2 - with complications). The nominal and continuous variables were compared. Results of the compar-

Table II. Laboratory results, nutritional and immunological parameters

\begin{tabular}{|c|c|}
\hline Parameter & Value \\
\hline Serum level of total protein $[\mathrm{g} / \mathrm{dl}]$ & $6.78 \pm 1.0(4.5-8.4)$ \\
\hline Serum level of albumin [g/dl] & $4.01 \pm 0.74(2.7-5.4)$ \\
\hline C-reactive protein (CRP) [mg/dl] & $4.46 \pm 4.59(0.45-20.82)$ \\
\hline Haemoglobin level (Hb) [g/dl] & $13.31 \pm 1.95(7.3-16.8)$ \\
\hline $\begin{array}{l}\text { White blood cell count (WBC) } \\
{\left[/ \mathrm{mm}^{3}\right]}\end{array}$ & $7.43 \pm 2.43(3.63-15.77)$ \\
\hline Total lymphocyte count [/mm³] & $1.73 \pm 0.71(0.76-3.62)$ \\
\hline Total lymphocyte count [\%] & $24.50 \pm 10.80(7.6-48.0)$ \\
\hline Total neutrophil count $\left[/ \mathrm{mm}^{3}\right]$ & $5.21 \pm 2.55(40.00-81.80)$ \\
\hline Total neutrophil count [\%] & $2.00 \pm 0.72(1.70-11.49)$ \\
\hline Total monocyte count [//mm $\mathrm{mm}^{3}$ & $0.76 \pm 0.48(0.32-2.41)$ \\
\hline Total monocyte count [\%] & $8.99 \pm 3.00(4.30-15.70)$ \\
\hline Platelet blood cell count [/mm³] & $259.42 \pm 104.49(99.00-621.00)$ \\
\hline $\begin{array}{l}\text { Neutrophil/lymphocyte ratio } \\
\text { (NLR) }\end{array}$ & $3.37 \pm 2.15(0.92-10.52)$ \\
\hline Platelet/lymphocyte ratio (PLR) & $154.36 \pm 60.04(60.00-398.08)$ \\
\hline $\begin{array}{l}\text { Lymphocyte/monocyte ratio } \\
(\text { LMR) }\end{array}$ & $2.88 \pm 1.45(0.48-6.00)$ \\
\hline Prognostic nutritional index (PNI) & $51.34 \pm 9.41(33.00-67.50)$ \\
\hline
\end{tabular}

Values are presented as means and standard deviations. 
ison are presented in Table IV and in Figure 1-4. Both groups were comparable according to age and gender distribution. The groups were comparable according to tumour location, splenectomy, and comorbidities. Both groups were comparable according to ASA classifications $(p=0.09)$. Although higher ASA classifications were more frequently noted in patients with complications compared to patients without complications. The median value of the intraoperative blood loss was higher in group 2, at $650 \mathrm{ml}$, than in group 1, at $400 \mathrm{ml}$, but the difference was not statistically significant $(p=0.15)$. The transfusion rate was comparable in both groups $(p=0.6)$. The significant differences in total and postoperative hospitalisation durations between both groups were noted. The median total hospitalisation duration was 10 days in group 1 versus 18.5 days in group 2 $(p=0.0018)$. The median postoperative hospitalisation duration was seven days in group 1 versus 16 days in group 2 ( $p=0.0008)$. The significant difference ( $p=$ 0.005 ) in NRS 2002 between both groups was noted. The higher NRS 2002 was noted in patients with postoperative complications (group 2) compared to patients without complications (group 1). Body mass index was comparable in both groups $(p=0.19)$. The median $\mathrm{BMI}$

Table III. Postoperative complications

\begin{tabular}{|c|c|}
\hline Complications & Value \\
\hline Pancreatic fistula/types: & $11(22.00 \%)$ \\
\hline A & $4(8.00 \%)$ \\
\hline B & $6(12.00 \%)$ \\
\hline C & $1(2.00 \%)$ \\
\hline Intraabdominal collection & $7(14.00 \%)$ \\
\hline Acute pancreatitis & $6(12.00 \%)$ \\
\hline Wound infection & $1(2.00 \%)$ \\
\hline Hydrothorax & $2(4.00 \%)$ \\
\hline Pneumonia & $1(2.00 \%)$ \\
\hline Pulmonary embolism & $1(2.00 \%)$ \\
\hline Intraabdominal bleeding & $1(2.00 \%)$ \\
\hline Splenic vein thrombosis & $1(2.00 \%)$ \\
\hline Relaparotomies/causes/postoperative day: & $2(4.00 \%)$ \\
\hline Retroperitoneal abscess (17 th day) & $1(2.00 \%)$ \\
\hline Intraabdominal bleeding ( $4^{\text {th }}$ day) & $1(2.00 \%)$ \\
\hline Rehospitalisations/causes/postoperative day: & $3(6.00 \%)$ \\
\hline Intraabdominal collections & $3(6.00 \%)$ \\
\hline Mortality rate & $0(0.00 \%)$ \\
\hline
\end{tabular}

Table IV. Comparison of groups without and with postoperative complications according to selected clinicopathological factors

\begin{tabular}{|c|c|c|c|}
\hline Parameter & $\begin{array}{c}\text { No } \\
\text { complications }\end{array}$ & Complications & $P$-value \\
\hline Age, mean [years] & 57.14 & 57.80 & $0.320^{a}$ \\
\hline \multicolumn{4}{|l|}{ Gender: } \\
\hline Male & $14(28 \%)$ & 7 (14\%) & $0.900^{b}$ \\
\hline Female & $21(42 \%)$ & $8(16 \%)$ & \\
\hline \multicolumn{4}{|l|}{ Location: } \\
\hline Tail & 5 & 5 & $0.500^{b}$ \\
\hline Trunk & 18 & 5 & \\
\hline Trunk and tail & 12 & 5 & \\
\hline \multicolumn{4}{|l|}{ Transfusion: } \\
\hline No & 32 & 13 & $0.600^{b}$ \\
\hline Yes & 3 & 2 & \\
\hline
\end{tabular}

\begin{tabular}{lccc}
\hline Splenectomy: & & & \\
\hline No & 5 & 5 & $0.250^{\mathrm{b}}$ \\
\hline Yes & 30 & 10 & \\
\hline NRS 2002: & & & \\
\hline 1 & 32 & 10 & $0.005^{\mathrm{b}}$ \\
\hline 2 & 1 & 4 & \\
\hline 3 & 1 & 4 & \\
\hline 4 & 0 & 1 & \\
\hline 5 & 0 & 0 & \\
\hline
\end{tabular}

\begin{tabular}{lccc}
\hline Arterial hypertension: & & & \\
\hline No & 24 & 9 & $0.790^{\mathrm{b}}$ \\
\hline Yes & 11 & 6 & \\
\hline
\end{tabular}

\begin{tabular}{lccc}
\hline Ischaemic heart disease: & & & \\
\hline No & 31 & 15 & $0.400^{\mathrm{b}}$ \\
\hline Yes & 4 & 0 & \\
\hline
\end{tabular}

\begin{tabular}{lccc}
\hline Diabetes mellitus: & & & \\
\hline No & 30 & 14 & $0.770^{b}$ \\
\hline Yes & 5 & 1 & \\
\hline
\end{tabular}

\begin{tabular}{lccc}
\hline Smoking: & & & \\
\hline No & 28 & 12 & $0.700^{b}$ \\
\hline Yes & 7 & 3 &
\end{tabular}

NRS 2002 - Nutritional Risk Score 2002. ${ }^{a}$ Student's t-test, ${ }^{b} \chi^{2}$ test. 
A

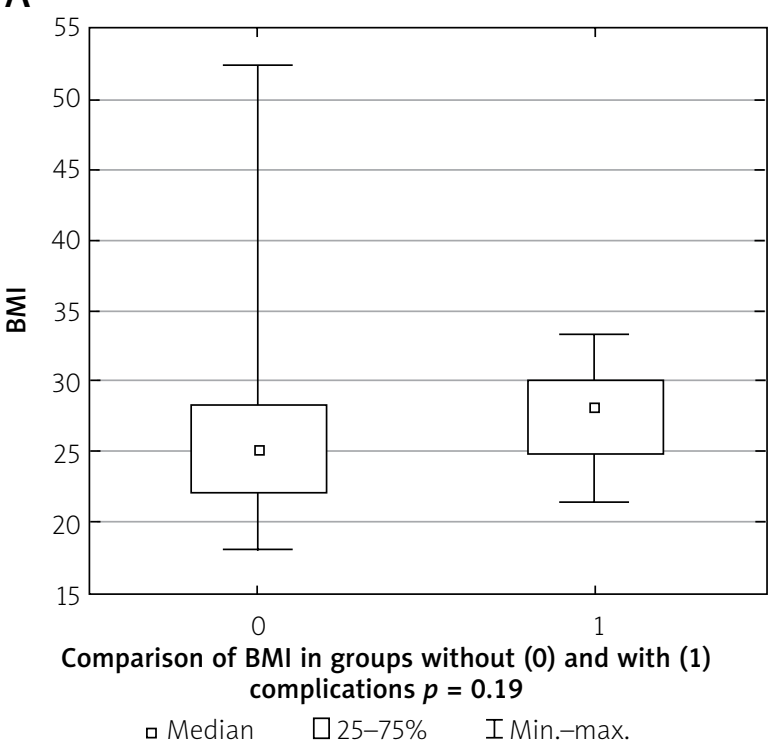

C

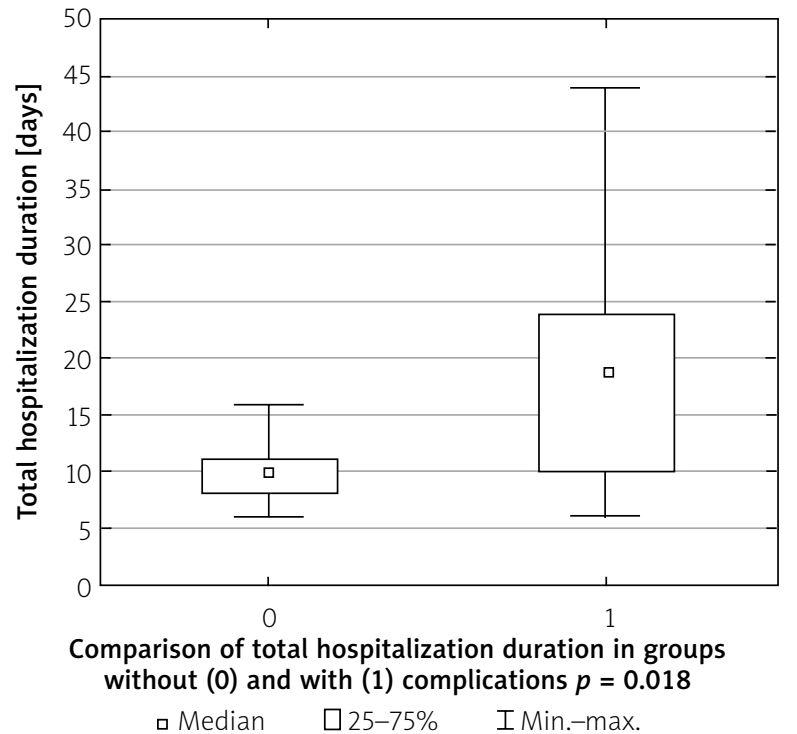

B

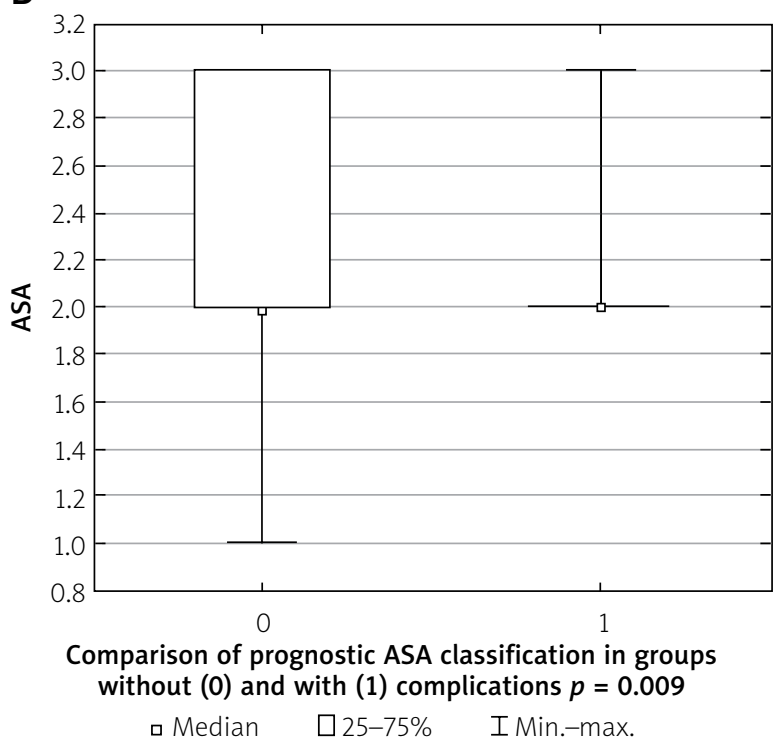

D

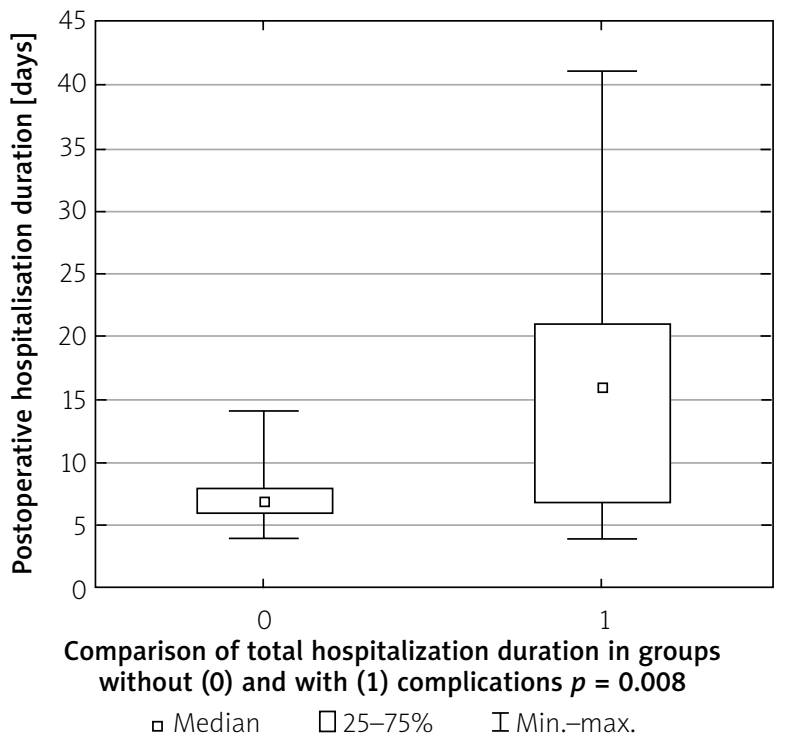

Figure 1. Comparison of BMI (A), ASA classification (B), total hospitalisation duration (C) and postoperative hospitalisation duration (D) between patients without complications (group 1) and with complications (group 2). Mann-Whitney $U$ test

was higher in group $2\left(28 \mathrm{~kg} / \mathrm{m}^{2}\right)$ compared to group 1 $\left(25 \mathrm{~kg} / \mathrm{m}^{2}\right)$. It should be noted that obesity was associated with higher risk of postoperative complications, but it was only a tendency, because the difference between both groups was not statistically significant. The total lymphocyte count was significantly higher in group 1 compared to group $2(p=0.001)$. The serum level of albumin was significantly higher in group 1 compared to group $2(p=0.0003)$. Also, the PNI was significantly higher in group 1 compared to group $2(p=0.0003)$. There was no significant difference in laboratory immu- nological parameters (NLR, LPR, LMR) in both groups. The level of C-reactive protein (CRP) was significantly higher in patients with complications compared to patients without complications (median value: 2 vs. $6 \mathrm{mg} /$ dl) $(p=0.03)$.

\section{Discussion}

In this study, we noted postoperative complications in $30 \%$ of patients. Pancreatic fistula was the most frequent complication in the analysed group. These reports are comparable with the literature data [20-23]. 
A

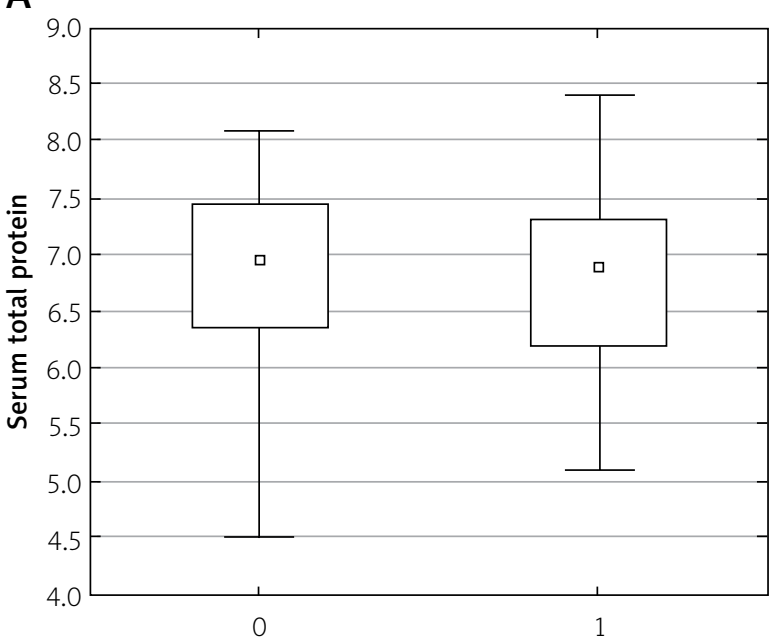

Comparison of serum total protein level in groups without (0) and with (1) complications $p=0.8620$

a Median $\quad \square 25-75 \% \quad$ I Min.-max.

\section{C}

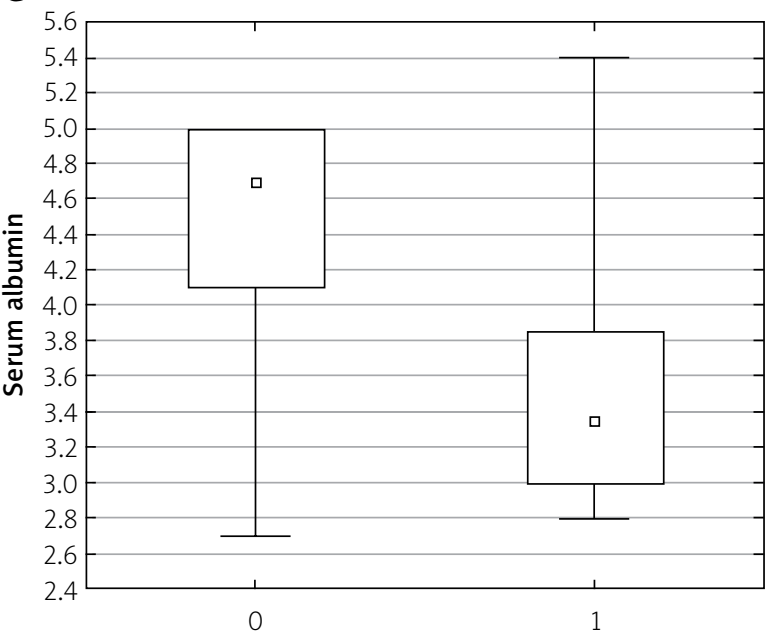

Comparison of serum albumin level in groups without (0) and with (1) complications $p=0.0003$

- Median $\quad \square 25-75 \% \quad$ IMin.-max.
B

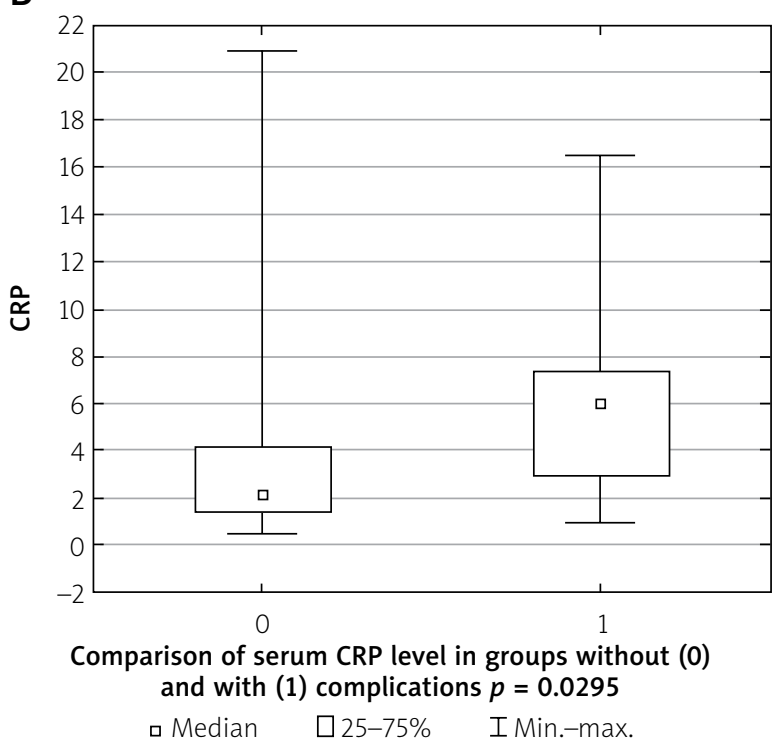

D

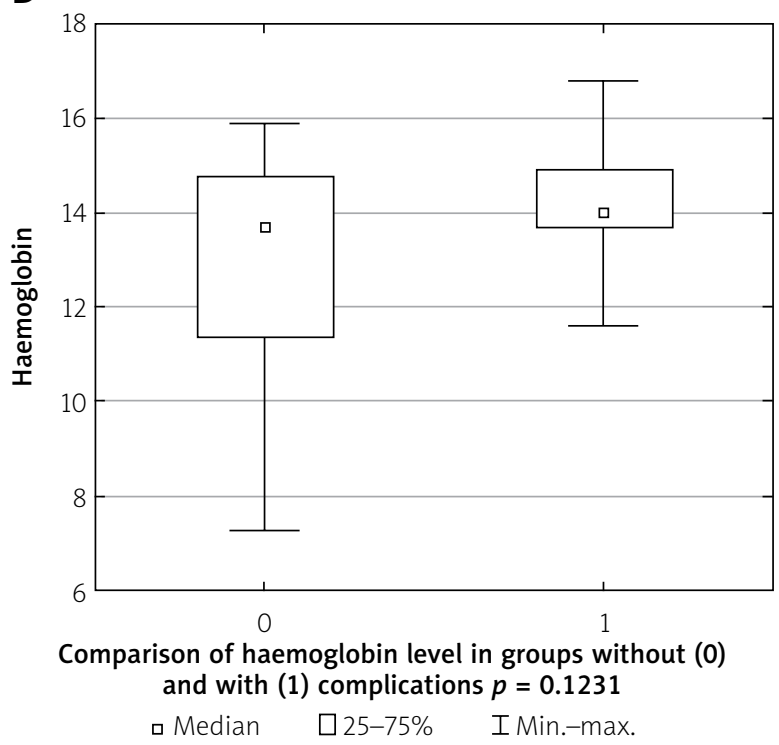

Figure 2. Comparison of serum total protein (A), C-reactive protein (CRP) (B), albumin (C), and haemoglobin (D) level between patients without complications (group 1) and with complications (group 2). Mann-Whitney $U$ test

It is known that malnutrition is associated with disturbances within humoral and cellular immune response, and it negatively influences healing of the postoperative wound. Additionally, malnutrition is associated with poor prognosis in patients due to a higher number of postoperative complications. Therefore, it is very important to assess the nutritional status before surgical treatment. The proper assessment of nutritional status allows the selection of malnourished patients and hence preoperative nutritional intervention in order to improve nutritional status and to decrease the risk of postoperative complications [10-15, 24].
In our study, blood test parameters including total protein, albumin, haemoglobin serum levels, and lymphocyte in the peripheral blood were analysed. Hypoalbuminaemia is associated with a higher number of postoperative complications following pancreatic resection due to impaired healing and activation of inflammatory response. The lymphocyte count in the peripheral blood is the second important laboratory parameter reflecting the immunological status and inflammatory response [15, 24-28].

Hendifar et al. [28] reported that higher preoperative albumin serum concentration was associated 
A

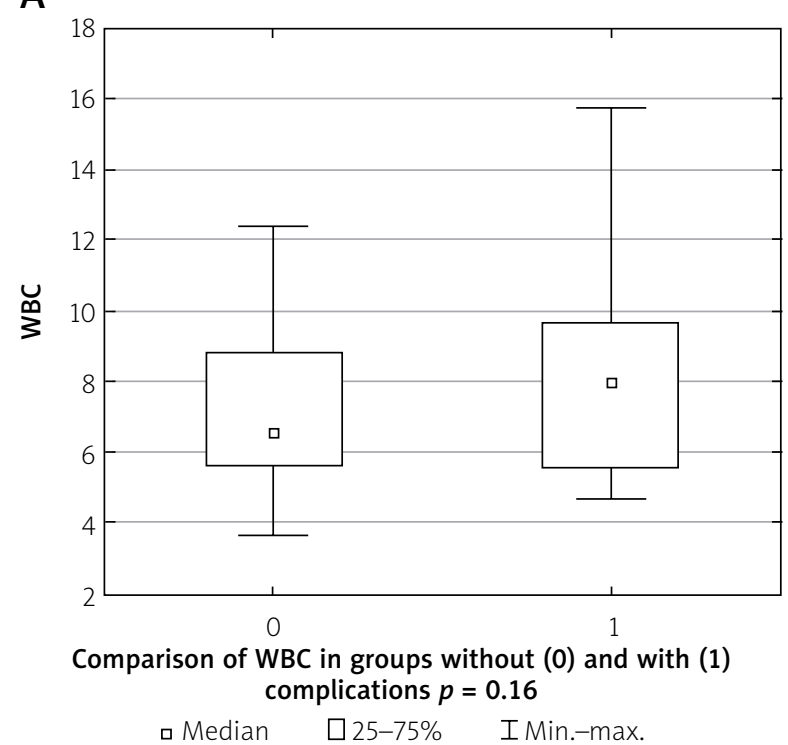

C

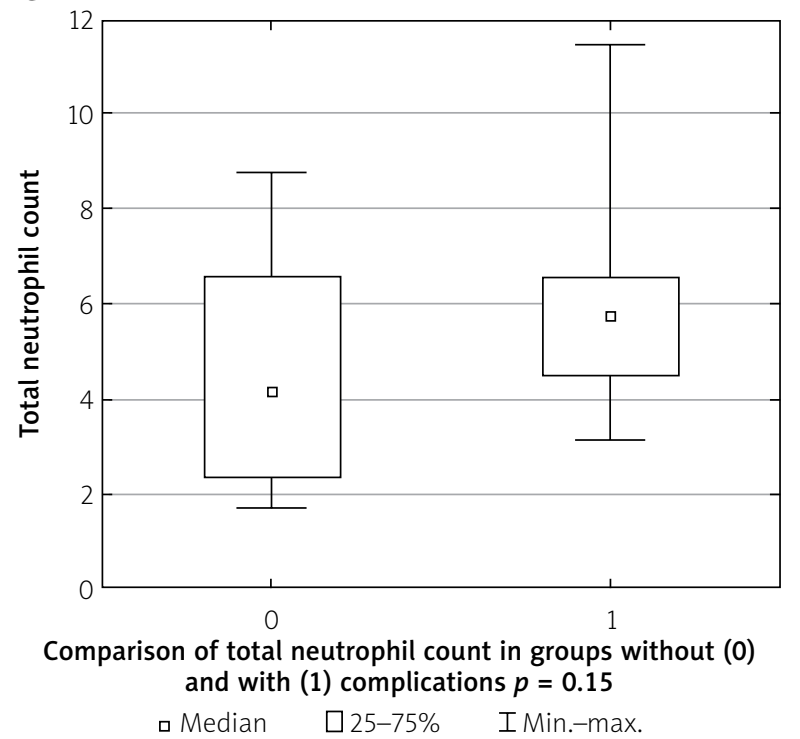

B

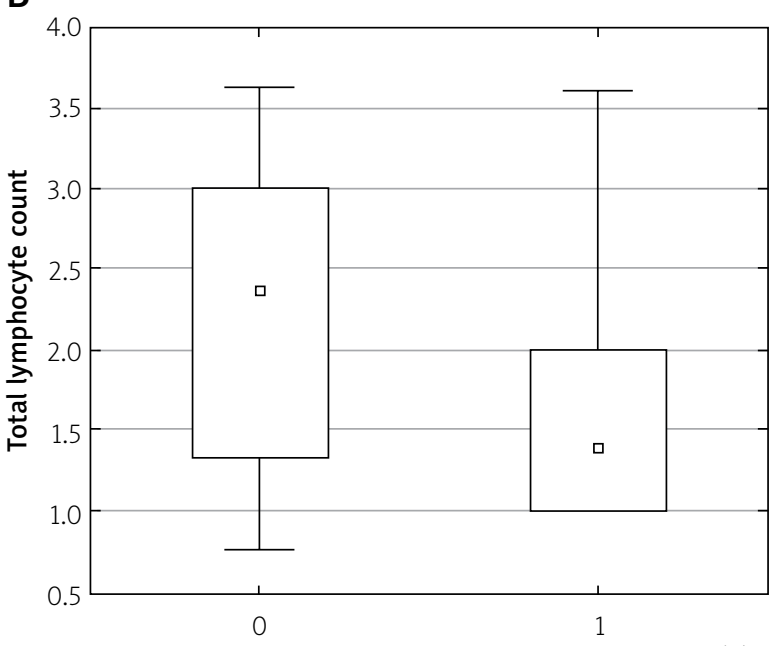

Comparison of total lymphocyte count in groups without (0) and with (1) complications $p=0.01$

- Median

$\square 25-75 \%$

I Min.-max.

D

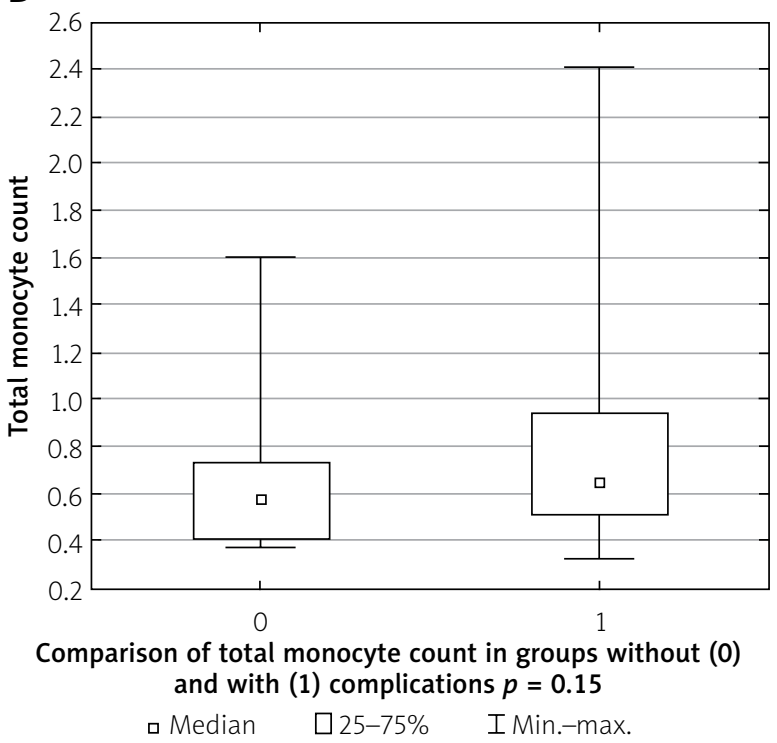

Figure 3. Comparison of WBC (A), total lymphocyte (B), neutrophil (C), and monocyte count (D) between patients without complications (group 1) and with complications (group 2). Mann-Whitney $U$ test

with greater overall survival in patients with resected pancreatic cancer. There was an association between lower level of albumin and transfusion rate and duration of hospitalisation [28]. In our study, an association between lower albumin serum level and greater incidence of postoperative complications was noted. The median serum albumin level was significantly lower in patients with postoperative complications compared to patients without complications. Additionally, a significantly lower total lymphocyte count was noted in patients with complications compared to patients without complications. The values of serum albumin and total lymphocyte count were used in order to calculate the PNI.

The PNI was described in 1980 by Buzby et al. [29] (their study included patients undergoing elective surgery) in order to assess immunological and nutritional aspects of patients undergoing surgery of the digestive tract [13, 24, 29]. In 1984, Onodera et al. proposed a modified PNI calculated on the basis of two factors: serum albumin and lymphocytes in the peripheral blood $[19,24]$. The authors observed that the incidence of postoperative complications was significantly higher in patients with low PNI. In 2014, Sun et al. [30] published 
A

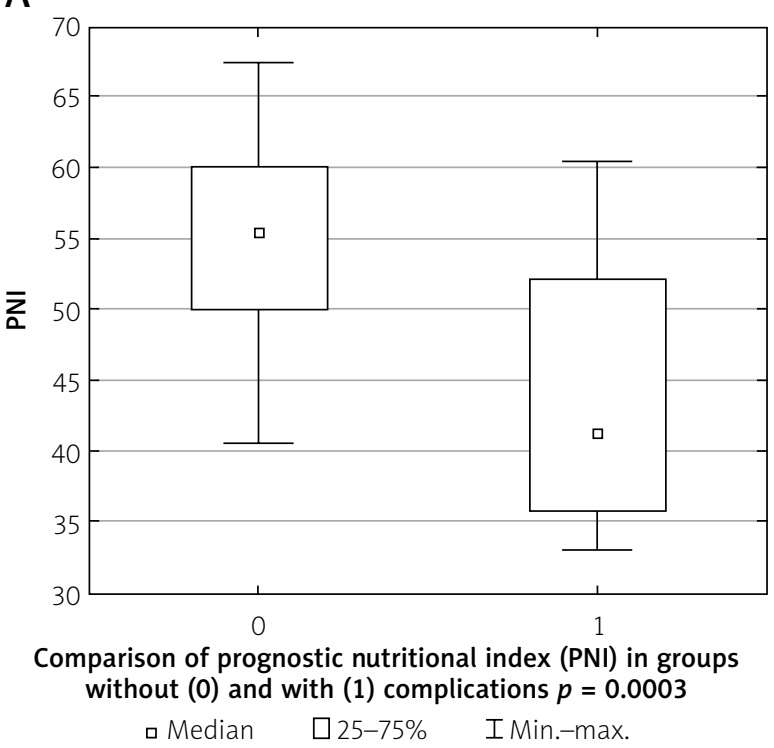

C

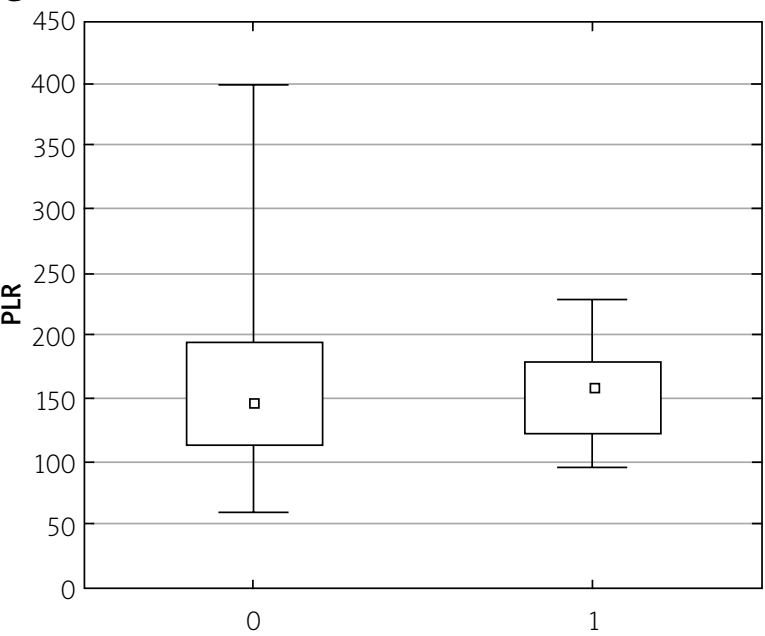

Comparison of platelet blood cell/lymphocyte ratio (PLR)

in groups without (0) and with (1) complications $p=0.69$ - Median $\quad$ 25-75\% IMin.-max.
B

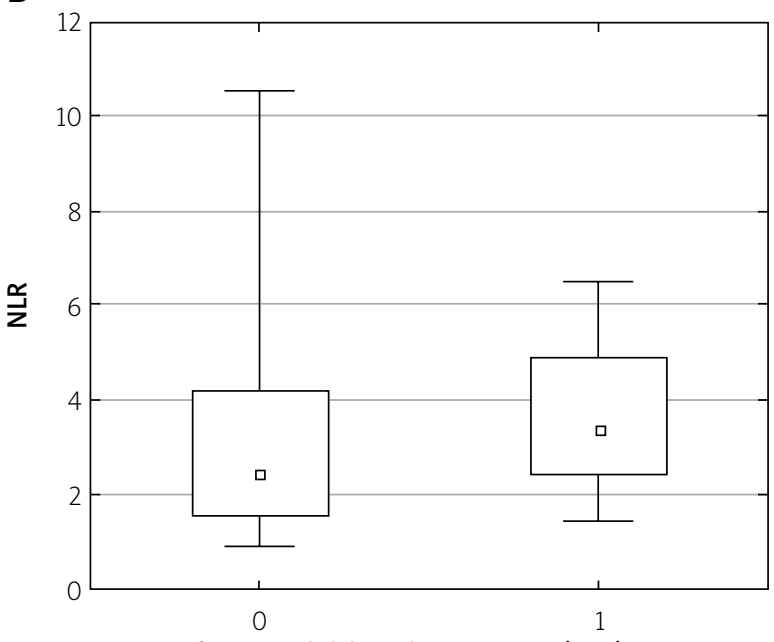

Comparison of neutrophil/lymphocyte ratio (NLR) in groups without (0) and with (1) complications $p=0.25$

- Median

$\square 25-75 \%$

I Min.-max.

D

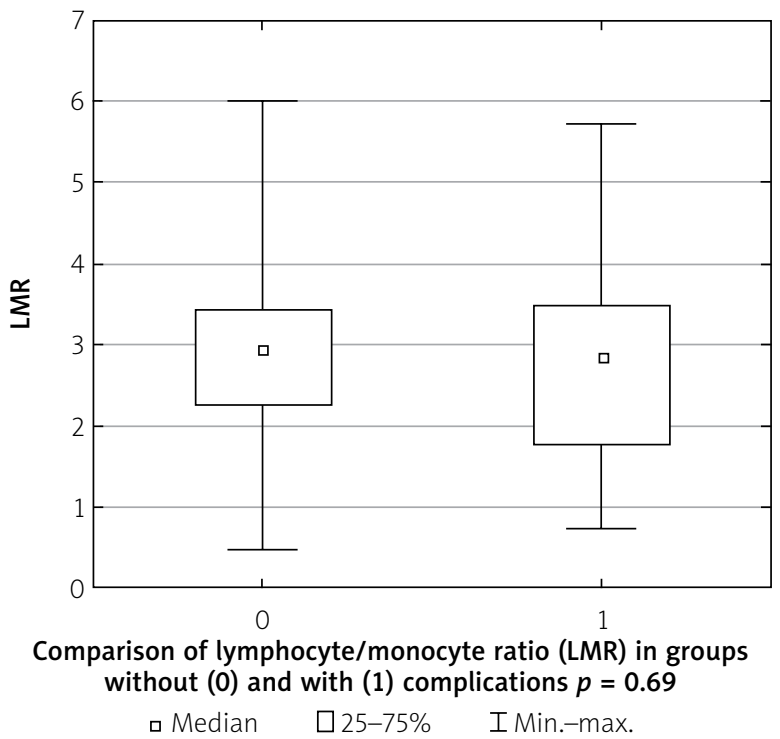

Figure 4. Comparison of prognostic nutritional index (PNI) (A), neutrophil/lymphocyte ratio (NLR) (B), platelet/lymphocyte ratio (PLR) (C) and lymphocyte/monocyte ratio (LMR) (D) between patients without complications (group 1) and with complications (group 2). Mann-Whitney $U$ test

a comprehensive meta-analysis regarding PNI. The article presented an analysis of 14 publications involving 3414 patients. The authors observed a significantly shorter overall survival and a higher number of postoperative complications in patients with low PNI but did not report a similar dependence for cancer-specific survival $[28,30]$. An association between lower PNI and higher number of postoperative complications was reported in our study, i.e. there was a significant difference in PNI values between patients without and with postoperative complications.
There are a lot of publications regarding the impact of prognostic nutritional index on postoperative outcome in patients following gastrointestinal surgery including oesophageal, gastric, colorectal, and pancreatic resections [9-15, 18, 19, 25-35].

Kanda et al. [34] analysed 268 patients undergoing pancreatectomy for pancreatic cancer. In multivariable analysis, low preoperative PNI (but not low albumin) was an independent prognostic factor for poor survival. Low preoperative albumin concentration and PNI were significantly associated with postoperative complica- 
tions. In this study, low PNI and low body mass index were associated with greater incidence of postoperative pancreatic fistula [34].

Sato et al. [35] analysed 44 patients undergoing distal pancreatectomy. The authors noted that rapid postoperative PNI reduction was associated with a greater incidence of postoperative pancreatic fistula. In this study, pancreatic fistula was noted in 23 (52\%) patients, of which 13 (56\%) were grade B or C [34].

Watanabe et al. [18] analysed inflammatory and nutritional parameters using the modified Glasgow Prognostic Score (mGPS), PNI, NLR, and PLR in 46 patients after pancreaticoduodenectomy for pancreatic cancer. In patients with $\mathrm{PNI}<40$, significantly lower haemoglobin levels and a higher number of intra-abdominal bleedings were noted. The postoperative pneumonia was more frequent in the mGPS 2 patients, and surgical complications greater than grade 3 (according to Clavien-Dindo classification) were significantly more frequent in the NLR $\geq 2.5$ patients. In multivariate analysis, only the PLR was an independent prognostic indicator. There are some other publications in the literature regarding the influence of immunological status on the incidence of postoperative complications [18].

An association between higher Nutritional Risk Screening NRS 2002 and greater number of postoperative complications was noted in this study. This study also revealed higher NLR and PLR in the complications group compared to the no complications group, but these differences were not statistically significant. Regarding inflammatory status, significantly higher CRP and lower LMR (no statistical difference) were noted in patients with complications compared to patients without complications, but these differences were not statistically significant.

In our study, the median BMI was higher in patients with complications $\left(28 \mathrm{~kg} / \mathrm{m}^{2}\right)$ compared to group without complications $\left(25 \mathrm{~kg} / \mathrm{m}^{2}\right)$. It should be noted that overweight or obesity were associated with higher risk of postoperative complications in our study, but it was only a tendency because the difference between both groups was not statistically significant. A review of the literature data shows that the influence of BMI on the incidence of postoperative complications in patients undergoing pancreatectomy is very interesting. In some studies (e.g. Kanda et al. [34]) lower BMI was independently associated with a higher risk of postoperative pancreatic fistula. In another study, high BMI was associated with a higher risk of postoperative complications, commonly pancreatic fistula. An association of low BMI with POPF may be caused by immunological dysfunction and impaired inflammatory response, and disturbances of the wound healing in underweight patients. Vanbrugghe et al. [36] analysed 208 patients following distal pancreatectomy. The authors reported that visceral obesity with $\mathrm{BMI} \geq 25 \mathrm{~kg} / \mathrm{m}^{2}$ was a significant risk factor for POPF. In this study, sarcopaenia did not influence the risk of clinically relevant pancreatic fistula. An association of high BMI with POPF may be caused by difficult intraoperative conditions and soft fat pancreas in overweight or obese patients. It has been proven that soft pancreas is a risk factor for POPF. Also, overweight and obesity are associated with a higher risk of infection complications. Generally obesity negatively influences major gastroenterological surgical procedures. It has been noted that obesity is associated with prolonged operative time and might be a risk factor for short-term complications; however, an adverse influence of obesity on long-term surgical outcomes was not observed [36-38].

There are also other studies regarding the influence of nutritional status on short-term outcome after pancreatectomy. The study by Kato et al. [39] conducted on 344 patients undergoing pancreatectomy for pancreatic cancer did not show an influence of nutritional status on postoperative complications. The correlations between the Controlling Nutritional Status score (CONUT) and postoperative complications were analysed. The authors concluded that nutritional status assessed as CONUT was associated with survival in patients with pancreatic cancer after pancreatectomy but was not associated with recurrence or postoperative complications [39]. The CONUT score is a scoring nutritional system based on calculation from the following three parameters: serum albumin level, total peripheral lymphocyte count, and total cholesterol level. It is categorised into normal nutrition status ( $0-1$ score), mild nutrition status (2-4 score), moderate nutrition status (5-8 score), and severe nutrition status (more than 8 score) [40]. In Kato's study, the CONUT score was not associated with postoperative pancreatic fistula, Clavien-Dindo grade, or postoperative duration of hospitalisation [39].

\section{Conclusions}

A review of the literature shows that there are a lot of reports regarding the impact of nutritional status on the incidence of postoperative complications in patients undergoing gastrectomy and colorectal surgery. In the field of pancreatic surgery, most studies focus on the influence of nutritional status on short-term outcome in patients following pancreaticoduodenectomy. There are a few reports on the influence of nutritional status in patients following distal pancreatectomy. Therefore, research in this field is needed and should be continued.

In conclusion, nutritional status significantly influences the incidence of postoperative complications in 
patients undergoing distal pancreatectomy. Assessment of nutritional status using PNI calculation should be the standard management of patients before surgical treatment.

\section{Acknowledgments}

This research received university grant number KNW-1-192/N/5/0.

\section{Conflict of interest}

The authors declare no conflict of interest.

\section{References}

1. Casadei R, Ricci C, Pezzilli R, et al. Assessment of complications according to the Clavien-Dindo classification after distal pancreatectomy. JOP J Pancreas 2011; 12: 126-30.

2. Simons JP, Shah SA, Ng SC, et al. National complication rates after pancreatectomy: beyond mere mortality. J Gastrointest Surg 2009; 13: 1798-805.

3. Yoshioka R, Saiura A, Koga R, et al. Risk factors for clinical pancreatic fistula after distal pancreatectomy: analysis of consecutive 100 patients. World J Surg 2010; 34: 121-5.

4. Fahy BN, Frey CF, Ho HS, et al. Morbidity, mortality, and technical factors of distal pancreatectomy. Am J Surg 2002; 183 237-41.

5. Balcom JH $4^{\text {th }}$, Rattner DW, Warshaw AL, et al. Ten-year experience with 733 pancreatic resections: changing indications, older patients, and decreasing length of hospitalization. Arch Surg 2001; 136: 391-8.

6. Olakowski M, Jabłońska B, Braszczok Ł, et al. Distal pancreatectomy: own experience. Pol Przegl Chir 2012; 84: 298-303.

7. Ryu SW, Kim IH. Comparison of different nutritional assessments in detecting malnutrition among gastric cancer patients. World J Gastroenterol 2010; 16: 3310-7.

8. Unsal D, Mentes B, Akmansu M, et al. Evaluation of nutritional status in cancer patients receiving radiotherapy: a prospective study. Am J Clin Oncol 2006; 29: 183-8.

9. Nozoe T, Ninomiya M, Maeda T, et al. Prognostic nutritional index: a tool to predict the biological aggressiveness of gastric carcinoma. Surg Today 2010; 40: 440-3.

10. Jiang N, Deng JY, Ding XW, et al. Prognostic nutritional index predicts postoperative complications and long-term outcomes of gastric cancer. World J Gastroenterol 2014; 20: 10537-44.

11. Lien YC, Hsieh CC, Wu YC, et al. Preoperative serum albumin level is a prognostic indicator for adenocarcinoma of the gastric cardia. J Gastrointest Surg 2004; 8: 1041-8.

12. Ray-Coquard I, Cropet C, Van Glabbeke M, et al. Lymphopenia as a prognostic factor for overall survival in advanced carcinomas, sarcomas, and lymphomas. Cancer Res 2009; 69: 5383-91.

13. Schwegler I, von Holzen A, Gutzwiller JP, et al. Nutritional risk is a clinical predictor of postoperative mortality and morbidity in surgery for colorectal cancer. Br J Surg 2010; 97: 92-7.

14. Bozzetti F, Gianotti L, Braga M, et al. Postoperative complications in gastrointestinal cancer patients: the joint role of the nutritional status and the nutritional support. Clin Nutr 2007; 26: 698-709.
15. Sun KY, Xu JB, Chen SL, et al. Novel immunological and nutritional-based prognostic index for gastric cancer. World J Gastroenterol 2015; 21: 5961-71.

16. Kondrup J, Allison SP, Elia M, et al. ESPEN guidelines for nutrition screening 2002. Clin Nutr 2003; 22: 415-21.

17. Kondrup J, Rasmussen HH, Hamberg O, Stanga Z. Nutritional risk screening (NRS 2002): a new method based on an analysis of controlled clinical trials. Clin Nutr 2003; 22: 321-36.

18. Watanabe J, Otani S, Sakamoto T, et al. Prognostic indicators based on inflammatory and nutritional status after pancreaticoduodenectomy for pancreatic cancer. Surg Today 2016; 46: 1258-67.

19. Onodera T, Goseki N, Kosaki G. Prognostic nutritional index in gastrointestinal surgery of malnourished cancer patients. Nihon Geka Gakkai Zasshi 1984; 85: 1001-5.

20. Bassi C, Dervenis C, Butturini G, et al.; International Study Group on Pancreatic Fistula Definition. Postoperative pancreatic fistula: an international study group (ISGPF) definition. Surgery 2005; 138: 8-13.

21. Malinka T, Klein F, Andreou A, et al. Distal pancreatectomy combined with multivisceral resection is associated with postoperative complication rates and survival comparable to those after standard procedures. J Gastrointest Surg 2018; 22: 1549-56.

22. van Hilst J, de Pastena M, de Rooij T, et al. Clinical impact of the updated international postoperative pancreatic fistula definition in distal pancreatectomy. HPB (Oxford) 2018; 20: 1044-50.

23. Pulvirenti A, Marchegiani G, Pea A, et al. Clinical Implications of the 2016 International Study Group on pancreatic surgery definition and grading of postoperative pancreatic fistula on 775 consecutive pancreatic resections. Ann Surg 2018; 268: 1069-75.

24. Brewczyński A, Jabłońska B, Pawlicki K. Associations between nutritional parameters and clinicopathologic factors in patients with gastric cancer: a comprehensive study. Nutr Cancer 2017; 69: 752-61.

25. Guo W, Ou G, Li X, et al. Screening of the nutritional risk of patients with gastric carcinoma before operation by NRS 2002 and its relationship with postoperative results. J Gastroenterol Hepatol 2010; 25: 800-3.

26. Hennessey DB, Burke JP, Ni-Dhonochu T, et al. Preoperative hypoalbuminemia is an independent risk factor for the development of surgical site infection following gastrointestinal surgery: a multi institutional study. Ann Surg 2010; 252: 325-9.

27. Li QQ, Lu ZH, Yang L, et al. Neutrophil count and the inflammation-based glasgow prognostic score predict survival in patients with advanced gastric cancer receiving first-line chemotherapy. Asian Pac J Cancer Prev 2014; 15: 945-50.

28. Hendifar A, Osipov A, Khanuja J, et al. Influence of body mass index and albumin on perioperative morbidity and clinical outcomes in resected pancreatic adenocarcinoma. PLoS One 2016; 11: e0152172.

29. Buzby GP, Mullen JL, Matthews DC, et al. Prognostic nutritional index in gastrointestinal surgery. Am J Surg 1980; 139: 160-7.

30. Sun K, Chen S, Xu J, et al. The prognostic significance of the prognostic nutritional index in cancer: a systematic review and meta-analysis. J Cancer Res Clin Oncol 2014; 140: 1537-49. 
31. Feng JF, Chen QX. Significance of the prognostic nutritional index in patients with esophageal squamous cell carcinoma. Ther Clin Risk Manag 2014; 10: 1-7.

32. Migita K, Takayama T, Saeki K, et al. The prognostic nutritional index predicts long-term outcomes of gastric cancer patients independent of tumor stage. Ann Surg Oncol 2013; 20: 2647-54.

33. Mohri Y, Inoue Y, Tanaka K, et al. Prognostic nutritional index predicts postoperative outcome in colorectal cancer. World J Surg 2013; 37: 2688-92.

34. Kanda M, Fujii T, Kodera Y, et al. Nutritional predictors of postoperative outcome in pancreatic cancer. Br J Surg 2011; 98: 268-74.

35. Sato N, Mori Y, Minagawa N, et al. Rapid postoperative reduction in prognostic nutrition index is associated with the development of pancreatic fistula following distal pancreatectomy. Pancreatology 2014; 14: 216-20.

36. Vanbrugghe C, Ronot M, Cauchy F, et al. Visceral obesity and open passive drainage increase the risk of pancreatic fistula following distal pancreatectomy. J Gastrointest Surg 2019; 23: 1414-24.

37. Jabłońska B, Lampe P, Dziki A, et al. The Association of Polish Surgeons on pancreatic fistulas. Pol Przegl Chir 2014; 86: 244-7.

38. Ri M, Aikou S, Seto Y. Obesity as a surgical risk factor. Ann Gastroenterol Surg 2017; 2: 13-21.

39. Kato Y, Yamada S, Suenaga M, et al. Impact of the controlling nutritional status score on the prognosis after curative resection of pancreatic ductal adenocarcinoma. Pancreas 2018; 47: 823-9.

40. Ignacio de Ulibarri J, Gonzalez-Madrono A, de Villar NG, et al. CONUT: a tool for controlling nutritional status. First validation in a hospital population. Nutr Hosp 2005; 20: 38-45.

Received: 28.05.2019

Accepted: 1.06.2019 